\title{
DESENVOLVIMENTO DE PLATAFORMA DE SIMULAÇÃO HARDWARE-IN-THE-LOOP DE BAIXO CUSTO
}

\author{
Aguinaldo Batista dos Santos Junior \\ Universidade de São Paulo, Escola Politécnica, Departamento de Engenharia Elétrica, PSI \\ E-mails: absantos@usp.br
}

\section{RESUMO}

A utilização de plataformas de simulação HIL (Hardware-in-the-loop) tem aumentado na indústria automotiva no processo de desenvolvimento de ECU's. A principal justificativa para tal aumento é a utilização da metodologia de desenvolvimento baseada em modelo, a qual integra a técnica HIL como um de seus principais pilares, possibilitando redução no tempo e custos de desenvolvimento de uma ECU. Quando aplicada a validação de sistemas eletrônicos de controle de freios, a simulação HIL possui vital importância, pois permite a prévia visualização de efeitos diretamente relacionados à dinâmica veicular, os quais eram somente analisados através da realização de testes em pistas. Neste artigo demonstra-se a criação de uma plataforma HIL de baixo custo de hardware para validação de uma ECU ABS. Esta plataforma é implementada incluindo o uso de um circuito elétrico para emulação de cargas e aquisição de sinais, de uma interface com entradas e saídas reconfiguráveis e de um computador de simulação, o qual executa os softwares de manipulação de dados e de simulação da dinâmica veicular. Os resultados obtidos através da comparação entre as simulações de um veículo com e sem a atuação do sistema ABS corroboram a operação da plataforma HIL desenvolvida.

\section{INTRODUÇÃO}

A crescente demanda por eficiência e conforto em veículos automotores teve como consequência direta o aumento do desenvolvimento de sistemas eletrônicos embarcados capazes de controlar diferentes tarefas ou funcionalidades de um automóvel.

Uma unidade de controle eletrônica (Electronic Control Unit, ou ECU) foi inicialmente aplicada em um veículo automóvel Chevy Cosworth Vega no ano de 1975 e consistia em um computador analógico o qual tinha a função de monitorar o motor e controlar a injeção de combustível nos cilindros [1].

No ano de 1981, a montadora de origem americana General Motors aplicou em toda sua produção um sistema micro controlado para gerenciamento do motor, o qual executava um código de 50.000 linhas [2].

A evolução da utilização de ECU's em veículos automotores ocorreu desde então de forma exponencial, colaborando para que outros sistemas fossem desenvolvidos e largamente 
aplicados em diferentes categorias de veículos automotores, como é o caso do sistema de freio antitravamento (Anti Lock Braking System, ou ABS).

Dentre as futuras inovações em sistemas veiculares, $90 \%$ serão baseadas no desenvolvimento de sistemas eletrônicos embarcados e dentre estas, $80 \%$ serão realizadas através de implementações de software [3]. Diante de tal cenário, ressalta-se a importância das atividades de validação dentro do processo de desenvolvimento de ECU's.

Este processo de validação é fundamental para que sejam atingidos os níveis requeridos de confiabilidade em um projeto de veículo automotor, tendo como principais benefícios a detecção prematura de desvios funcionais e implementação mais estável de hardware e software.

Falhas neste processo podem representar um grande impacto negativo para um fabricante de automóveis, resultando em perdas financeiras as quais envolvem altos custos de reparo, encargos legais e danos à imagem corporativa da montadora. Exemplo disso ocorreu em 2014 com determinado fabricante automotivo do mercado asiático que convocou o recall global de 1,9 milhões de automóveis de um dos seus modelos devido a uma grave falha de software [4].

Com o objetivo de reduzir ou mesmo eliminar possíveis falhas em ECU's, as montadoras de veículos automotores têm empregado diferentes técnicas destinadas à validação do projeto. Esse processo deve possuir características que atendam aos requisitos de robustez, eficiência, confiabilidade e custo/benefício.

Neste contexto, a metodologia conhecida como desenvolvimento baseado em modelos (model based design, ou MBD) tem se tornado cada vez mais popular nas indústrias automotivas. De acordo com Santos e Neme [5], a principal característica desta é o desenvolvimento em uma plataforma única, que permite a criação da planta do sistema e de seu controlador utilizandose de uma mesma ferramenta computacional. Dentro da perspectiva de validações, esta plataforma proporciona a identificação e correção de erros de maneira integrada ao desenvolvimento, tendo como consequências diretas as reduções nos custos e tempo de lançamento de um produto no mercado.

Dentre as validações executadas na metodologia MBD destaca-se a simulação HIL, a qual é utilizada para realização de simulações em tempo real, dentro das quais se incluem modelos matemáticos que representam o processo ou a planta controlada, assim como, um componente real para o qual se deseja realizar a validação.

A importância deste tipo de simulação aumenta quando ela é aplicada em sistemas que utilizam informações dinâmicas de um veículo automotor em determinadas condições de rodagem, como por exemplo, o sistema ABS de freios ou o sistema de controle de estabilidade. Dependendo do sistema, informações tais como: velocidades das rodas, ângulo de direção, aceleração lateral e rotação em torno do próprio eixo vertical, são utilizadas no algoritmo de controle da ECU e coletadas através de sensores distribuídos pelo veículo.

Durante o processo de desenvolvimento de uma ECU que utiliza tais informações, diversas validações veiculares de hardware e software seriam necessárias, aumentando os custos e o tempo de desenvolvimento de um projeto. No entanto, as validações através do uso de HIL e softwares de simulação veicular permitem que grande parte dos testes necessários sejam 
executados em bancada, eliminando-se, por exemplo, a necessidade de coleta de dados em pistas de prova e subsequente correção de algoritmos de controle.

Não somente as indústrias automotivas são beneficiadas através do uso das plataformas HIL. Outros segmentos industriais como o de equipamentos médicos, geração de energia, automação industrial e aeroespacial também se utilizam desta técnica no desenvolvimento de projetos. Isso se deve a redução nos custos de construção de sistemas HIL. Anteriormente estes poderiam variar entre US\$ $50.000,00$ e US\$ $1.000 .000,00$, porém com a redução do custo dos computadores e aumento da capacidade de processamento, atualmente, tais sistemas podem ser adquiridos por valores na faixa de US\$ 6.000,00 [6].

\section{OBJETIVO}

O objetivo deste trabalho é desenvolver uma plataforma HIL com baixo custo de hardware, para testes e validações de um módulo eletrônico ABS.

\section{PROCESSO DE DESENVOLVIMENTO DE ECU'S}

O número de módulos eletrônicos em veículos automotores tem aumentado à medida que surgem novas demandas de mercado ou mesmo devido a novas legislações. Mesmo os veículos básicos podem ser equipados com um número que varia entre 30 a 50 ECU's para o controle de diferentes funcionalidades e sistemas, o que significa a execução de milhares de linhas de código de software [2].

Diante deste cenário, é de fundamental importância que os fabricantes apliquem métodos eficientes e inovadores no desenvolvimento de ECU's, colaborando para redução nos custos e tempo de desenvolvimento associados a esta atividade.

Neste contexto, a metodologia MBD tem se apresentado como uma alternativa eficiente e de alta aceitação por parte dos fabricantes. Dentre seus principais objetivos destacam-se [5]:

- O desenvolvimento de modelos baseados em requisitos;

- Criação de códigos de forma automática;

- Otimização de algoritmos através de simulação;

- Documentação de projeto produzida de forma contínua;

- Contínua identificação e correção de falhas através da integração dos testes com o desenvolvimento.

Esta metodologia emprega técnicas sequenciais de validação da função de software. Estas são conhecidas como: MIL (Model-in-the-loop), SIL (Software-in-the-loop) e HIL (Hardware-inthe-loop). A figura 1 apresenta as fases do MBD e como cada uma das técnicas de validação está integrada dentro deste processo. 


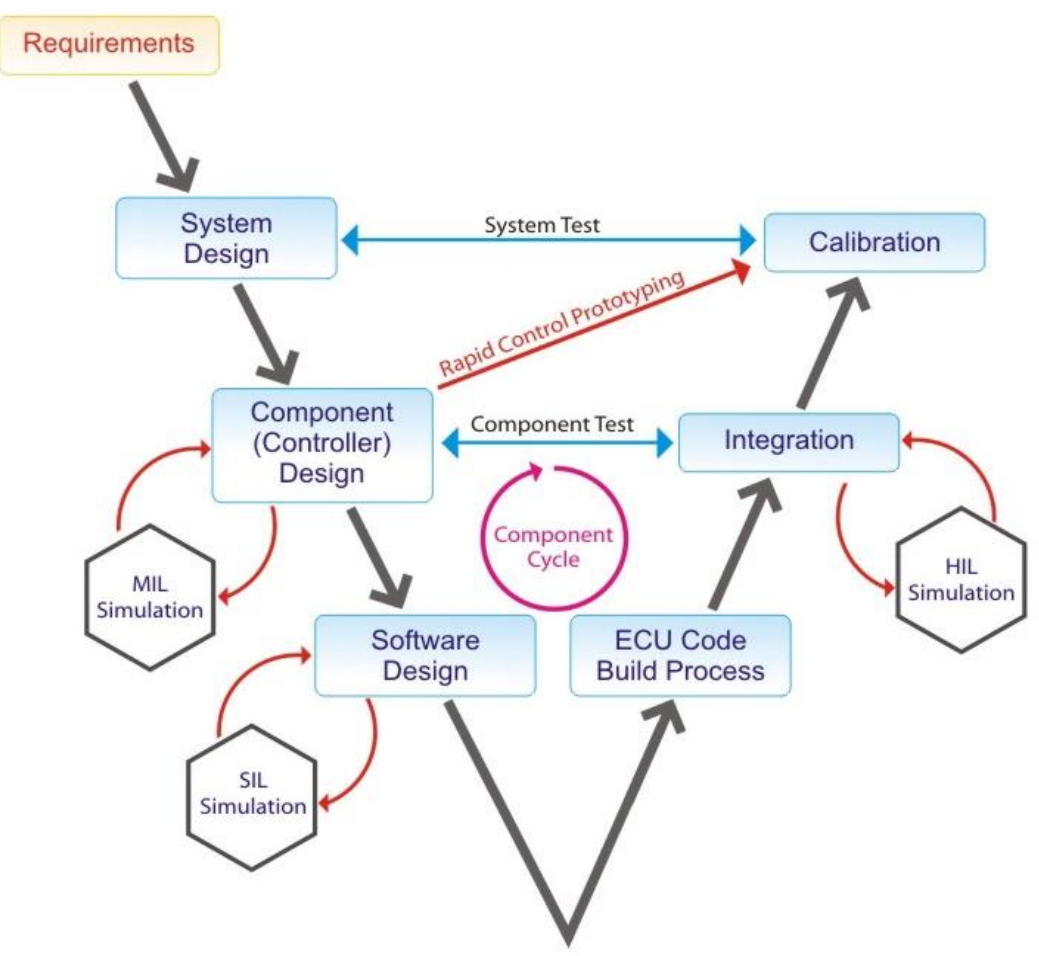

FIGURA 1. Desenvolvimento baseado em modelo

FONTE: SRM Technologies. Model Based Development. Em:

< http://209.200.79.65/automotive/automotive.htm >. Acesso em: 22 Maio 2016.

De acordo com Gühmann [7], o desenvolvimento de uma ECU é iniciado através das especificações do cliente, as quais são geralmente elaboradas em forma de um caderno de requisitos.

A próxima etapa do processo consiste no design do sistema, sendo que os modelos dos controladores são desenvolvidos em ambientes de programação gráficos. Através da simulação MIL, os modelos dos controladores são testados com o objetivo de verificar se atendem aos requisitos do projeto.

A etapa seguinte consiste no teste e otimização dos modelos das funções utilizando ferramentas apropriadas de hardware e software. Através da prototipagem rápida, por exemplo, o modelo de software é verificado diretamente em um veículo utilizando-se de um computador by-pass.

A simulação MIL e a prototipagem rápida permitem a identificação e a correção de falhas em um estágio inicial do processo de desenvolvimento.

Em seguida, na etapa de design de software, o modelo anteriormente utilizado é substituído pelo código fonte e software base que serão utilizados na ECU. Neste caso, as validações são realizadas através de simulação SIL, na qual o modelo da planta e software são executados em uma mesma máquina.

Assim que o software é embarcado no módulo eletrônico, realiza-se a validação e teste do componente através da simulação HIL, na qual se conecta o hardware da ECU a um sistema 
em tempo real que simula a planta controlada. Nesta fase, o objetivo é encontrar falhas no sistema operacional e em componentes de hardware utilizados na construção do módulo eletrônico. O processo de calibração de parâmetros e curvas da ECU também é comumente realizado com o suporte da simulação HIL.

\subsection{Simulação HIL}

A simulação HIL pode ser definida como uma técnica na qual um sistema em malha fechada é emulado através do uso de componentes reais e simulados. Ela se apresenta como uma das últimas etapas no processo de validação e teste de uma ECU, subsequente aos testes MIL e SIL, com a diferença de que nesta fase os modelos de software são convertidos para uso no hardware específico da ECU em desenvolvimento.

A figura 2 apresenta o princípio básico de operação de um sistema HIL. Utilizando-se de um computador de simulação, as informações de entrada necessárias ao sistema testado são geradas pelo modelo da planta e enviadas a um simulador de sensores. Este tem a função de emular características elétricas dos sensores e transformar as informações em sinais que são interpretados pelo sistema em teste.

A emulação de todos os sinais elétricos necessários para o pleno funcionamento da ECU e a execução em tempo real do modelo virtual da planta são pré-requisitos para que a simulação HIL seja executada com sucesso. Caso a emulação de sinais não seja completamente atendida, o módulo eletrônico pode não efetuar o processamento das informações, pois entende que se encontra em condição de falha.

Após o processamento, os sinais de resposta do sistema testado são adquiridos e realimentados ao modelo da planta, formando um sistema em malha fechada.

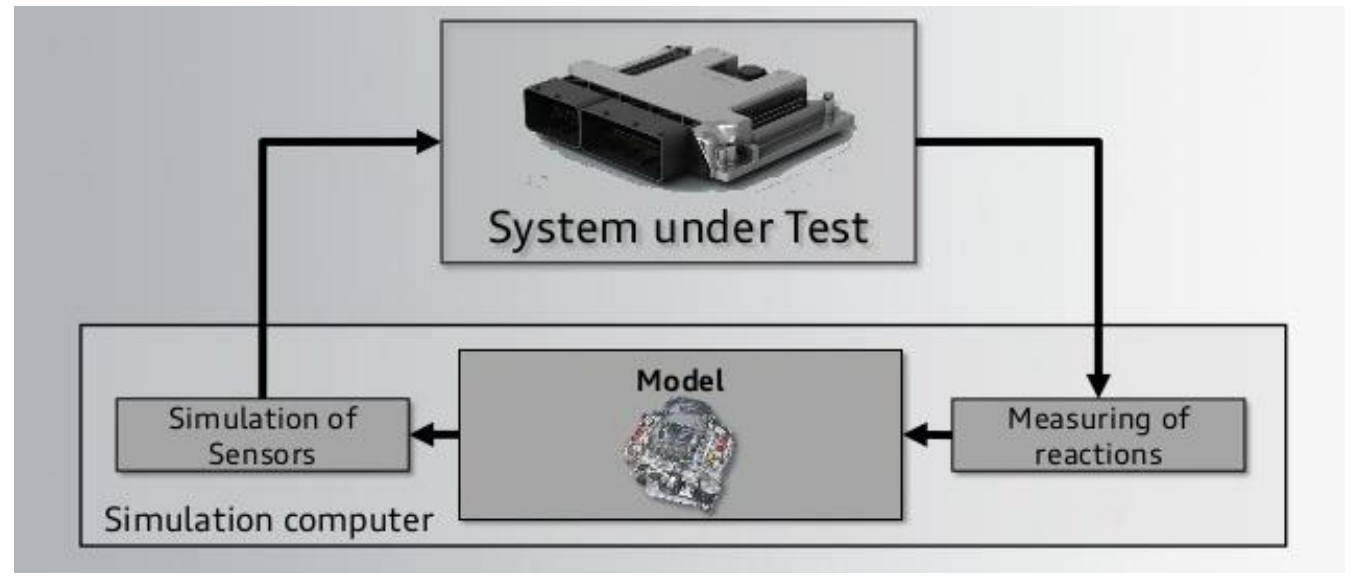

FIGURA 2. Princípio básico de operação de um sistema HIL

FONTE: AUDI AG. Application of DDS on Modular Hardware-in-the-loop test benches at Audi. Em: < http://pt.slideshare.net/RealTimeInnovations/2014-10-08rtiddsuserconferencefina >. Acesso em: 22 Maio 2016.

A simulação HIL é considerada como uma das técnicas mais seguras para validação de componentes reais em ambientes virtuais e por isso é largamente aplicada no processo de desenvolvimento de ECU's. Uma das suas aplicações típicas relaciona-se ao 
desenvolvimento de ECU's ABS, sendo possível a realização de testes em ambiente simulado, evitando o uso de veículos reais em pistas de teste e reduzindo os riscos de acidentes.

De acordo com Fathy [8], alguns fatores devem ser considerados na implementação de um sistema HIL conforme abaixo:

- Acuracidade e fidelidade das informações referentes aos sensores e atuadores utilizados no sistema;

- Condicionamento e processamento digital de sinais utilizados na simulação. Um exemplo disso está relacionado aos sinais provenientes de sensores, os quais normalmente precisam ser condicionados através do uso de filtros;

- Processadores de alto desempenho e sistemas operacionais de tempo real. Uma vez que as interações entre componentes físicos e virtuais de um simulador HIL são bidirecionais é crucial que os eventos estejam exatamente sincronizados no tempo, exigindo com que os componentes virtuais da simulação sejam executados em tempo real;

- Diagnóstico avançado para dispositivos físicos utilizados na simulação HIL, como por exemplo, sensores;

- Os modelos virtuais utilizados na simulação devem atender dois requisitos básicos para que o sistema possa atingir seu objetivo. O primeiro requisito exige que o modelo deva ser capaz de representar precisamente a dinâmica da planta para a qual se deseja realizar a simulação. $\mathrm{O}$ segundo requisito exige que o modelo utilizado possa ser executado em tempo real;

- Integração Multithreading e Multirate. Um problema comumente encontrado em simulações HIL é a rigidez do modelo virtual, a qual pode ser definida como a disparidade entre as velocidades características dos diferentes componentes do modelo virtual. Modelos rígidos podem ser encontrados em diferentes áreas, mas particularmente em mecatrônica são facilmente notáveis devido as diferentes velocidades de resposta de componentes elétricos e mecânicos. Quando essas diferenças não podem ser eliminadas através de técnicas de modelagem é comum que a dinâmica destes componentes seja integrada através de diferentes taxas, o que é conhecido como Multirate Integration. Esta integração pode ser realizada através do uso de um único processador via Multithreading, porém é comumente executada através do uso de múltiplos processadores;

- Distribuição dos componentes físicos e virtuais de um simulador HIL dentro de uma rede. Alguns benefícios podem ser mencionados para o uso de componentes de uma simulação em um contexto de rede: os componentes de hardware necessários para a simulação podem não estar localizados no mesmo espaço físico e, portanto o uso de uma rede pode auxiliar neste aspecto; a distribuição de componentes virtuais em uma rede pode colaborar para o aumento da capacidade de processamento na simulação HIL, uma vez que as informações não são processadas por um único processador; a capacidade de interligar em rede os diferentes componentes de um simulador HIL torna possível a criação de simuladores independentes para diferentes sistemas, o que possibilita a combinação entre eles e a formação de um amplo sistema HIL;

- Integração entre hardware e software. Com o objetivo de se obter um simulador HIL eficiente, a sinergia entre o protótipo de hardware e o modelo virtual também deve ser um fator considerado. Isso pode ser atingido através do particionamento, o que significa dividir um sistema em subsistemas constituídos de protótipos de hardware e 
componentes simulados através da tecnologia HIL. Neste caso é importante considerar a causalidade de conexão entre eles, ou seja, como será o fluxo dos sinais entre estes subsistemas.

\section{METODOLOGIA DE PESQUISA}

No presente trabalho aplicou-se a abordagem de pesquisa quantitativa utilizando-se de simulação.

A tabela 1 apresenta cada um dos componentes utilizados no desenvolvimento da plataforma e suas respectivas funções.

Tabela 1. Componentes utilizados na plataforma desenvolvida e respectivas funções

\begin{tabular}{|l|l|}
\hline Componente & Função \\
\hline ECU ABS & Sistema testado \\
\hline Circuito elétrico & Emulação de cargas e aquisição de sinais \\
\hline $\begin{array}{l}\text { Interface de entradas e saídas } \\
\text { reconfiguráveis }\end{array}$ & Entrada e saída de dados \\
\hline \multirow{4}{*}{ Computador de simulação } & Modelo matemático do veículo \\
\cline { 2 - 2 } & Interface com modelo matemático do veículo \\
\cline { 2 - 2 } & Cálculo das frequências dos sinais dos sensores \\
\cline { 2 - 2 } & Cálculo da variação de pressão nos cilindros de freio \\
\hline
\end{tabular}

A figura 3 apresenta a arquitetura física do sistema proposto.
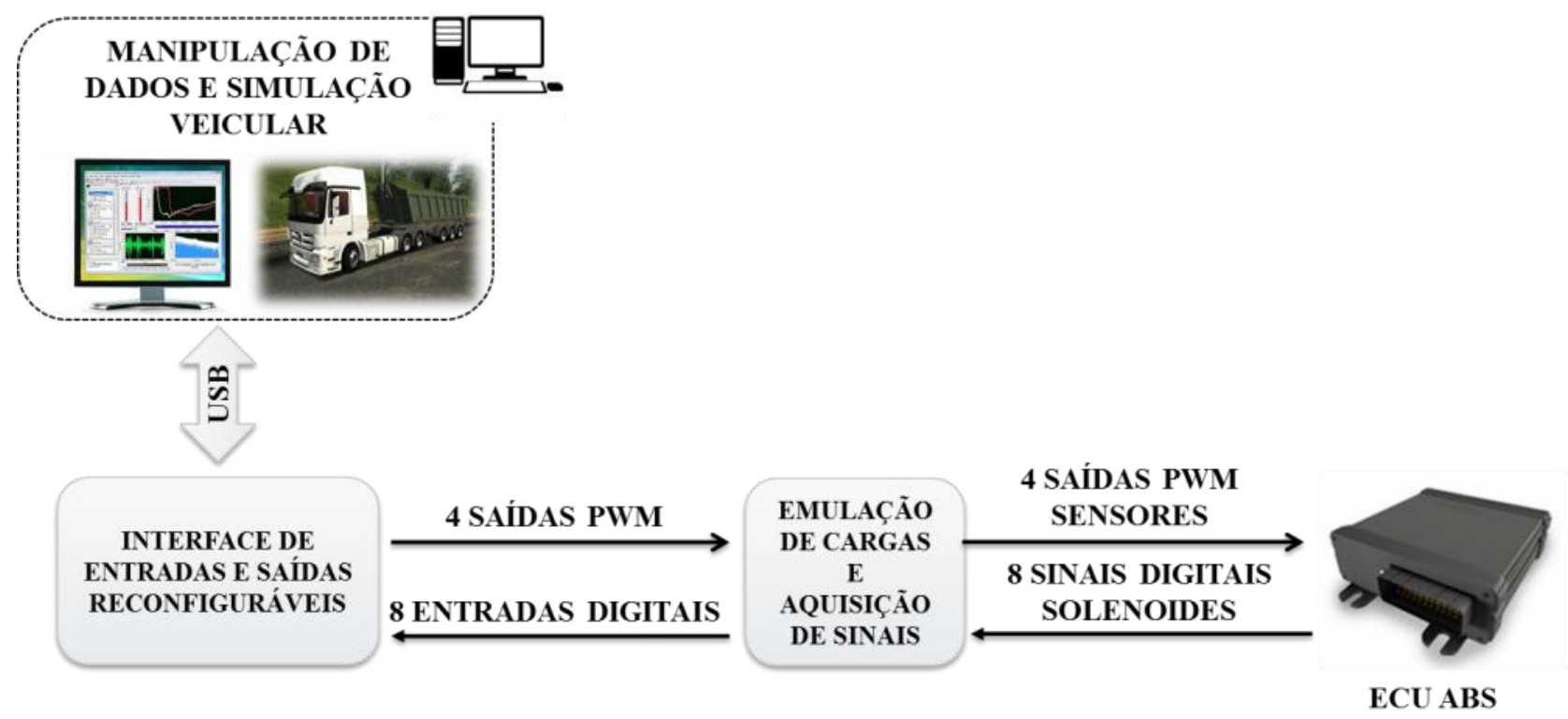

FIGURA 3. Arquitetura da plataforma HIL desenvolvida FONTE: O próprio autor

A seguir são apresentados os detalhes de implementação da plataforma HIL. 


\subsection{ECU ABS}

A ECU ABS utilizada nesta implementação é aplicada em veículos comerciais com sistema pneumático e opera na arquitetura $4 \mathrm{~S} / 4 \mathrm{M}$ (quatro sensores de velocidade de roda e quatro válvulas moduladoras).

Os sensores utilizados em veículos comerciais são do tipo passivo. Estes são compostos por um ímã permanente conectado a uma haste de metal em torno da qual uma bobina é enrolada. Um anel dentado montado no cubo de roda é responsável por induzir uma tensão alternada na bobina do sensor à medida que cada dente passa pela extremidade dele.

A impedância, a tensão de saída e o sinal de ruído devem ser compatíveis com os requisitos da ECU ABS. Para o módulo em teste neste trabalho, o manual de manutenção determina que a impedância do sensor deva ter valores entre 900 e 2000 $\Omega$. Caso esta condição não seja atendida, a ECU entra em condição de falha e não opera corretamente.

A válvula moduladora de pressão é responsável por controlar a pressão do ar sobre o freio de uma determinada roda durante uma intervenção ABS. Internamente, ela é constituída por duas válvulas diafragma, as quais são controladas por dois solenoides. A ECU possui 8 saídas as quais são responsáveis pela atuação de cada solenoide.

As válvulas também possuem impedância característica a qual deve ser compatível com a ECU ABS. Para o módulo em teste neste trabalho, o manual de manutenção determina que a impedância de cada um dos solenoides deva ter valores entre 4 e $9 \Omega$. Caso esta condição não seja atendida, a ECU entra em condição de falha e não opera corretamente.

Para que tais condições fossem atendidas, foi necessária a criação de um circuito elétrico para emulação dos sinais e cargas conectados a ECU.

\subsection{Circuito elétrico emulador de cargas e sinais}

Para que as condições de funcionamento da ECU ABS fossem atendidas foi necessária a construção de um circuito elétrico para emulação de cargas e sinais.

Foram utilizadas 4 resistências de $1 \mathrm{k} \Omega$ para emulação da impedância dos sensores de velocidade das rodas. A figura 4 apresenta a conexão entre a interface de entradas e saídas reconfiguráveis e a ECU ABS.

Da mesma forma, foram utilizadas 8 resistências de $8,2 \Omega$ para emulação da impedância dos solenoides das válvulas moduladoras. No entanto, como as saídas da ECU enviam um sinal de $12 \mathrm{~V}$ e as entradas digitais da interface suportam uma tensão máxima de $5 \mathrm{~V}$, foi necessária a inclusão de um circuito adicional para tratamento do sinal, conforme apresentado na figura 5. Este circuito é composto por 2 fotoacopladores de quatro canais, aplicados com o objetivo de isolar a tensão de $12 \mathrm{~V}$ proveniente da ECU, da tensão de 5V utilizada na interface de entradas e saídas 
reconfiguráveis. Adicionalmente, este circuito transfere o sinal elétrico correspondente ao estado das válvulas para a interface de entradas e saídas reconfiguráveis.

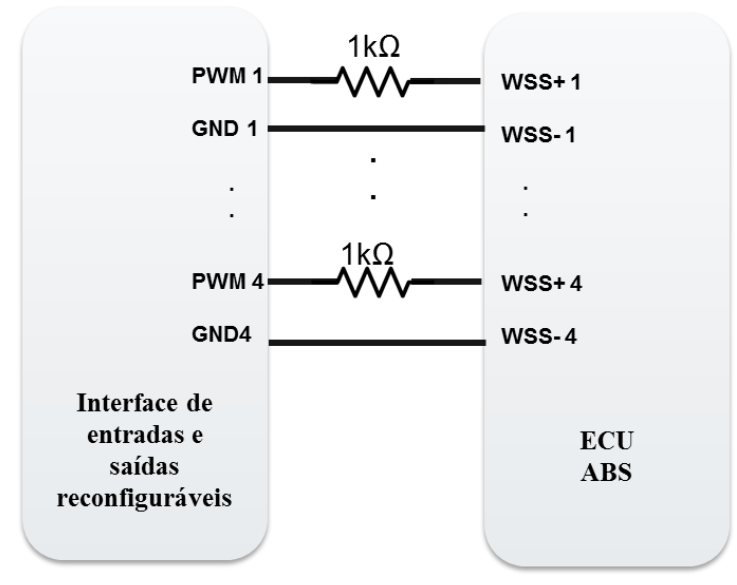

FIGURA 4. Emulação da impedância dos sensores de velocidade FONTE: O próprio autor

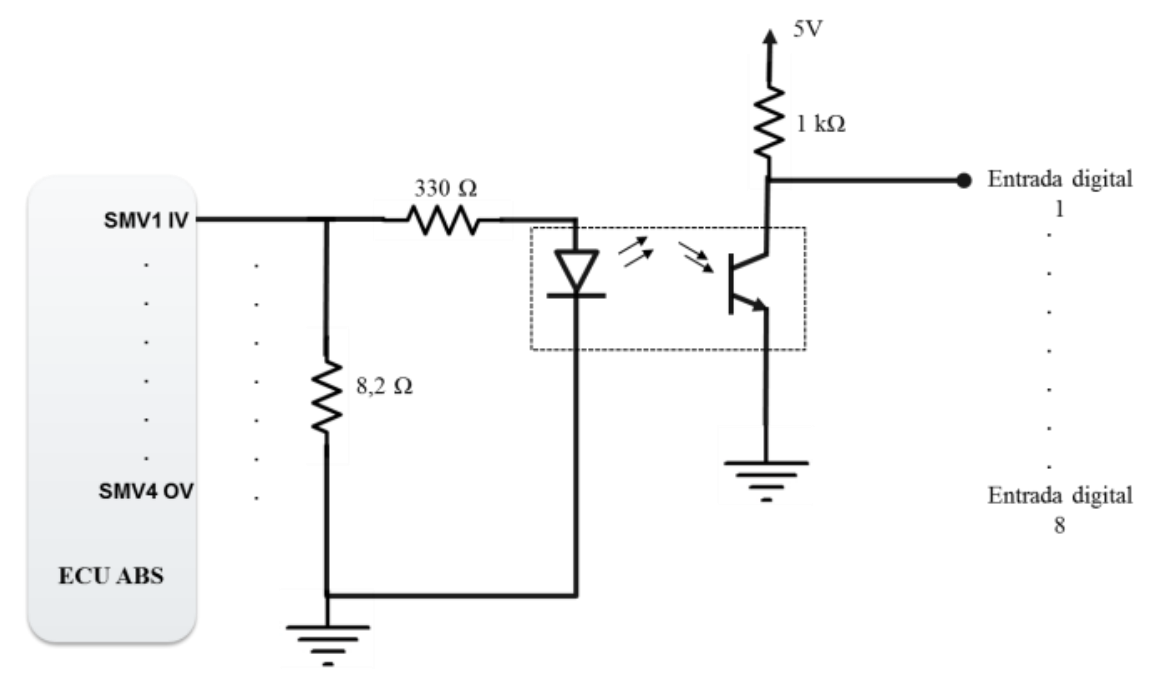

FIGURA 5. Emulação da impedância dos solenoides FONTE: O próprio autor

3.3. Interface de entradas e saídas reconfiguráveis

Foram utilizadas 4 saídas PWM disponíveis na interface de entradas e saídas reconfiguráveis para gerar os sinais dos sensores de velocidade das rodas.

A aquisição do estado de cada um dos solenoides das válvulas moduladoras foi realizada através de 8 entradas digitais.

\subsection{Computador de Simulação}

O computador de simulação utilizado neste trabalho possui um processador de $1,7 \mathrm{GHz}$ com dois núcleos e memória RAM de 8GB. 
Neste computador foram executados os softwares a seguir.

\subsubsection{Software de simulação veicular}

O software de simulação veicular utilizado nos testes dispõe de modelos matemáticos precisos de veículos comerciais permitindo a manipulação de suas características. Além disso, proporciona a análise da dinâmica veicular o que facilita a visualização dos efeitos de uma frenagem utilizando o sistema ABS.

Outro fator importante é que o software permite a manipulação de características do ambiente da simulação, como por exemplo, o coeficiente de atrito da pista.

Vale ressaltar que a plataforma HIL é capaz de operar com diferentes softwares de simulação veicular desde que estes contenham modelos matemáticos precisos e que sejam capazes de operar em tempo real.

\subsubsection{Interface com modelo matemático do veículo}

A interface com o modelo matemático do veículo foi implementada através de uma DLL. Conforme apresentado na figura 6, é possível selecionar quais variáveis são importadas e exportadas do modelo.

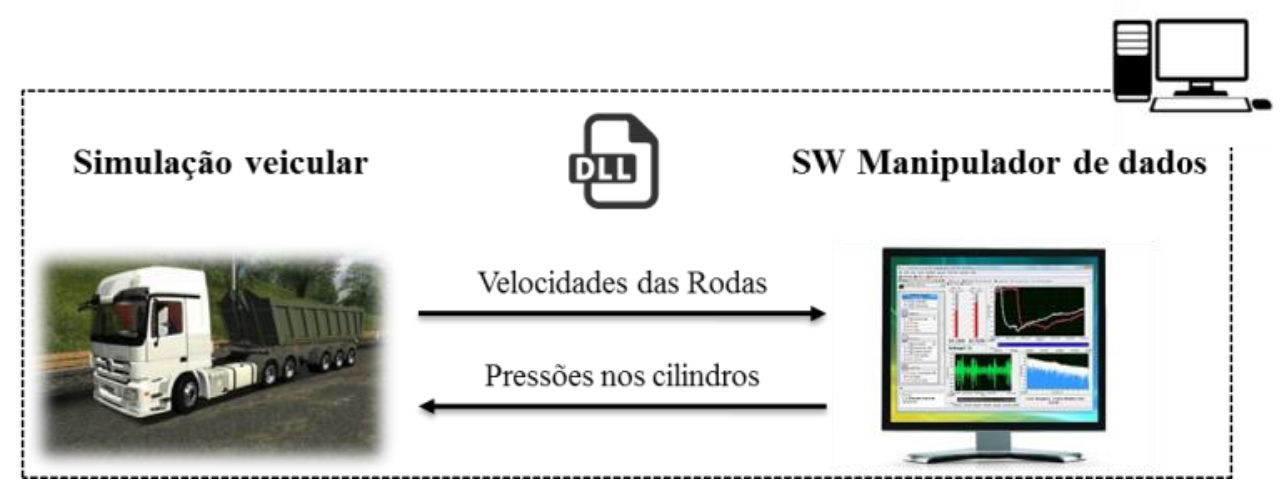

FIGURA 6. Interface entre software de simulação veicular e manipulador de dados

FONTE: O próprio autor

\subsubsection{Cálculo da frequência do sinal dos sensores}

Os sensores de velocidade das rodas do tipo passivo geram um sinal AC com característica senoidal e que aumenta em amplitude e frequência de maneira diretamente proporcional à velocidade das rodas. A figura 7 apresenta as características típicas de um sinal gerado por este tipo de sensor. 


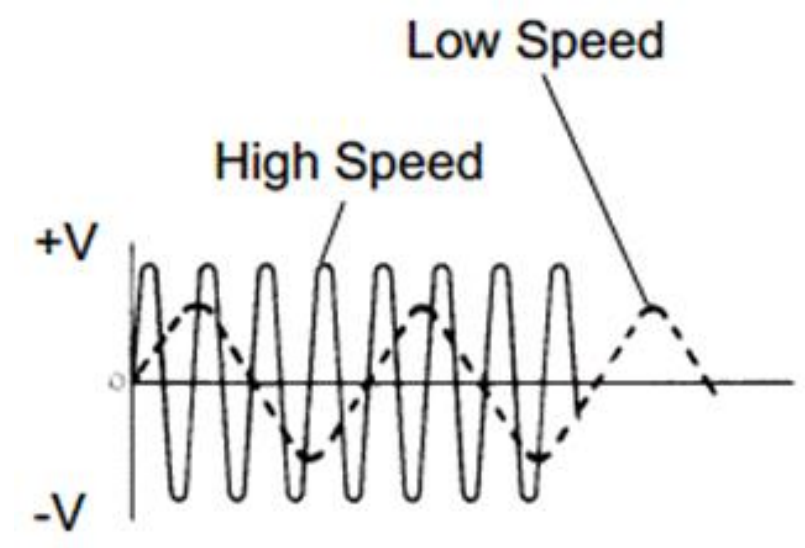

FIGURA 7. Sinal gerado por um sensor de velocidade do tipo passivo FONTE: AUTOCODES. Wheel Speed Sensor Signal. Em: <http://www.autocodes.com/c0205_toyota.html >. Acesso em: 22 Maio 2016.

Conforme mencionado anteriormente, saídas PWM foram utilizadas para gerar o sinal de velocidade das rodas. O uso destes sinais somente foi possível após a realização de medições com a ECU ABS.

Através do uso de um gerador de sinais, a ECU ABS foi estimulada com formas de onda senoidal e quadrada em diferentes amplitudes, frequências e níveis médios. A leitura dos valores calculados pela ECU foi realizada através do CAN J1939 (mensagem EBC2, identificador 18 FE BF 0B), disponível no módulo.

Mesmo com diferentes parâmetros de tipo de sinal, amplitude e nível médio, a ECU somente apresentou variação na leitura da velocidade quando houve variação da frequência do sinal. Tais resultados são apresentados na tabela 2.

De acordo com Tobro e Magnusson [9], a seguinte relação para cálculo velocidade em função da frequência é válida:

$$
v_{v}=\frac{o_{v}}{N} \cdot f_{s}
$$

Nela $v_{v}$ representa a velocidade de uma roda, $o_{v}$ o perímetro do pneu, $N$ o número de dentes do anel dentado utilizado na roda e $f_{s}$ a frequência do sinal.

Nas medições realizadas, a ECU ABS possuía parâmetros de perímetro de pneu de 3,075 m e número de dentes do anel dentado igual a 100. Aplicando-se estes valores na equação 1 e efetuando o cálculo para cada uma das frequências mencionadas na tabela 2 , é possível observar que os resultados obtidos possuem pequenas diferenças àqueles obtidos no ensaio, com erro máximo de $3,458 \%$. 
Tabela 2. Levantamento de frequência $x$ velocidade calculada pela ECU ABS

\begin{tabular}{|c|c|c|c|}
\hline Frequência $(\mathbf{H z})$ & Velocidade $(\mathbf{k m} / \mathbf{h})$ - Ensaio & Velocidade $(\mathbf{k m} / \mathbf{h})$ - Calculada & Erro \% \\
\hline 100 & 10,7 & 11,07 & 3,458 \\
\hline 200 & 22,3 & 22,14 & 0,717 \\
\hline 300 & 32,8 & 33,21 & 1,250 \\
\hline 400 & 44,3 & 44,28 & 0,045 \\
\hline 500 & 55,3 & 55,35 & 0,090 \\
\hline 600 & 66,6 & 66,42 & 0,270 \\
\hline 700 & 77,3 & 77,49 & 0,246 \\
\hline 800 & 88,5 & 88,56 & 0,068 \\
\hline 900 & 99,6 & 99,63 & 0,030 \\
\hline 1000 & 111,2 & 110,7 & 0,450 \\
\hline 1100 & 122,5 & 121,77 & 0,596 \\
\hline 1200 & 133,2 & 132,84 & 0,270 \\
\hline
\end{tabular}

Baseando-se nestas informações, foi implementado através de software o cálculo da frequência dos sinais PWM enviados a ECU ABS.

\subsubsection{Cálculo da variação de pressão nos cilindros de freio}

Uma vez que o sistema proposto não utiliza válvulas moduladoras reais, foi estabelecido um equacionamento para o cálculo das pressões de frenagem em cada uma das rodas.

Conforme mencionado anteriormente, cada válvula moduladora possui 2 solenoides, os quais modificam o modo de operação do sistema de acordo com sua atuação. A tabela 3 apresenta os possíveis estados de uma válvula moduladora.

Tabela 3. Modos de operação de uma válvula moduladora ABS

\begin{tabular}{|c|c|c|}
\hline Modo de operação & Solenoide A & Solenoide B \\
\hline Modo inativo & Inativo & Inativo \\
\hline Modo reduzir & Ativo & Ativo \\
\hline Modo manter & Inativo & Ativo \\
\hline
\end{tabular}

Caso ambos os solenoides estejam inativos, a pressão do freio de serviço é transmitida diretamente ao cilindro de freio. Portanto, foi necessário estabelecer uma relação matemática que reflita a transmissão da pressão do freio de serviço ao cilindro de freio através da válvula moduladora. Esta transmissão se dá a uma taxa $k_{\text {build }}$, a qual foi determinada neste projeto de forma empírica através de simulações com a ECU ABS. Neste caso, adotou-se $k_{\text {build }}=10 \mathrm{bar} / \mathrm{s}$. Portanto, tem-se que:

$$
\begin{gathered}
\Delta p=p_{f s}-p_{0} \\
p_{1}=p_{0}+\Delta p * k_{\text {build }}
\end{gathered}
$$


A diferença $(\Delta p)$ entre a pressão no freio de serviço $\left(p_{f s}\right)$ e a pressão atual no cilindro de freio $\left(p_{0}\right)$ é multiplicada pela taxa de transmissão de pressão $\left(k_{\text {build }}\right)$. Este valor é então somado a pressão atual no cilindro de freio $\left(p_{0}\right)$, resultando no valor de pressão incrementado $\left(p_{1}\right)$. Através deste modelo o aumento de pressão no cilindro de freio ocorre em degraus, uma vez que a execução dos ciclos do software é realizada a cada $1 \mathrm{~ms}$.

Caso ambos os solenoides estejam ativos, a entrada de pressão de ar através da válvula moduladora é interrompida e a saída para exaustão é liberada fazendo com que ocorra a redução da pressão no cilindro. A redução de pressão de ar pela válvula moduladora se dá a uma taxa $k_{\text {release }}$, a qual foi determinada neste projeto de forma empírica através de simulações com a ECU ABS. Neste caso adotou-se $k_{\text {release }}=40 \mathrm{bar} / \mathrm{s}$. Portanto, tem-se que:

$$
\begin{gathered}
\Delta p=p_{0}-p_{\text {atm }} \\
p_{1}=p_{0}-\Delta p * k_{\text {release }}
\end{gathered}
$$

A diferença $(\Delta p)$ entre a pressão no cilindro $\left(p_{0}\right)$ e a pressão atmosférica $\left(p_{a t m}\right)$ é multiplicada pela taxa de decréscimo de pressão $\left(k_{\text {release }}\right)$. Este valor é então subtraído da pressão atual no cilindro de freio $\left(p_{0}\right)$, resultando no valor de pressão reduzido $\left(p_{1}\right)$. Através deste modelo a redução de pressão no cilindro de freio ocorre em degraus, uma vez que a execução dos ciclos do software é realizada a cada $1 \mathrm{~ms}$.

Caso somente o solenoide B esteja ativo, a entrada de pressão de ar e a saída para exaustão são interrompidas, fazendo com que a pressão existente no cilindro seja mantida. Portanto, tem-se que:

$$
p_{1}=p_{0}
$$

\subsection{Custos de implementação da plataforma}

O custo dos hardwares utilizados para a implementação da plataforma desenvolvida foi de aproximadamente $\$ 2.100,00$. Estes incluem a interface de entradas e saídas reconfiguráveis, circuito para emulação de cargas e aquisição de sinais e um computador de alta capacidade de processamento para execução dos softwares de simulação veicular e manipulação de dados.

O custo relativo ao software de simulação veicular não foi considerado devido a grande diversidade de opções e preços disponíveis no mercado. Porém, dada à flexibilidade da plataforma HIL, qualquer uma destas opções poderia ser utilizada desde que atendam aos requisitos descritos no item 3.4.1.

Da mesma forma, o custo da ECU ABS não foi considerado por se tratar do sistema testado. 


\section{ANÁLISE DOS RESULTADOS}

A integração dos componentes anteriormente apresentados permitiu a concepção da plataforma HIL para testes de uma ECU ABS. A figura 8 apresenta de forma integrada o fluxo de dados através dos componentes utilizados nesta plataforma.

As indicações em azul apresentam o fluxo dos dados referentes às velocidades das rodas. Estas são geradas através do modelo matemático e após o processo de tratamento dos sinais são recebidas pela ECU ABS.

As indicações em verde apresentam o fluxo de dados referentes ao estado dos solenoides de cada uma das válvulas moduladoras. Estas informações são geradas pela ECU ABS e após a manipulação por software são transformadas em valores de pressão nos cilindros de freio. Estes valores são, então, recebidos pelo modelo matemático.

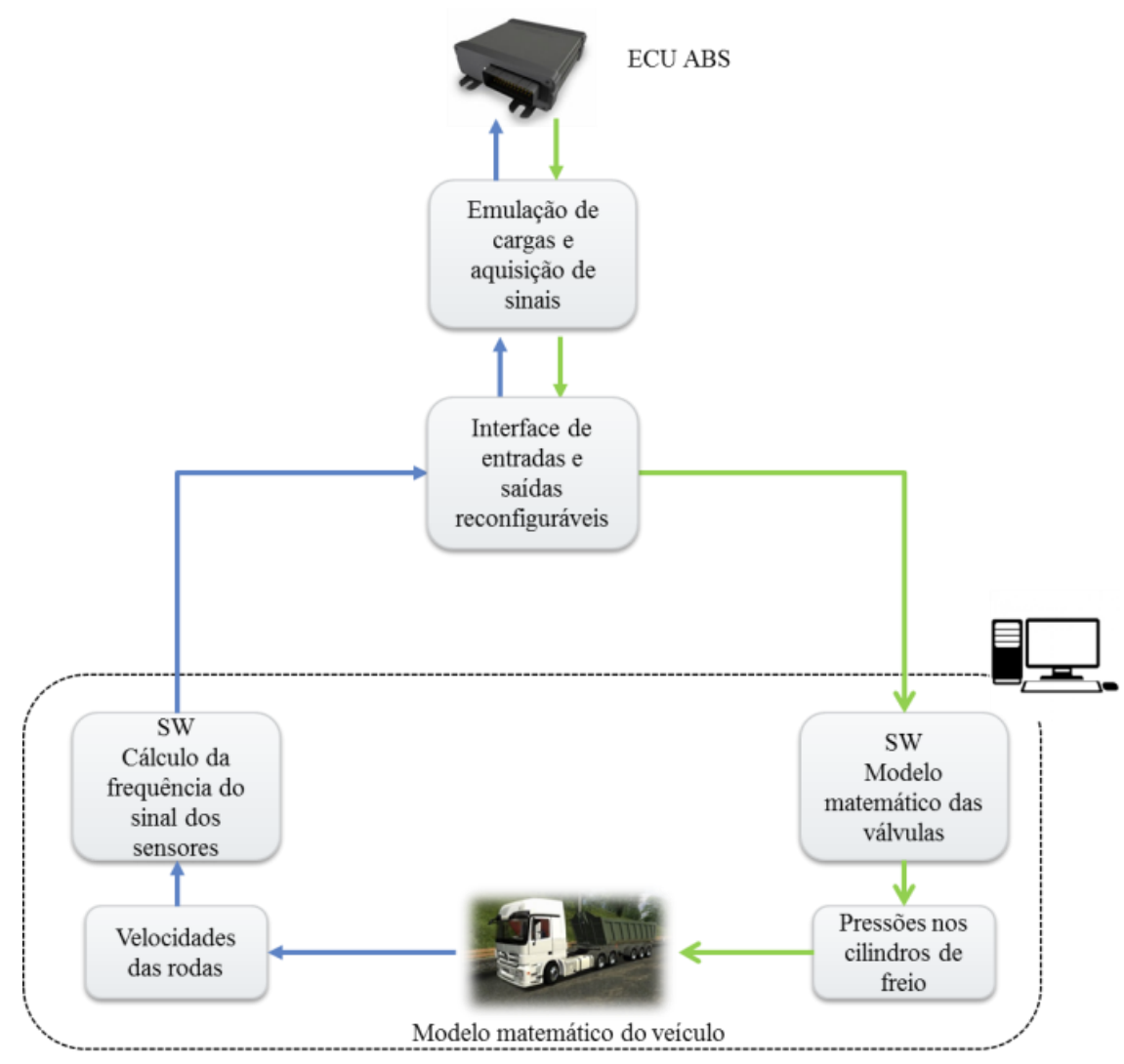

FIGURA 8. Fluxo de dados entre os componentes da plataforma HIL FONTE: O próprio autor

As simulações para verificação do funcionamento do sistema foram realizadas com um caminhão $6 \times 2$ de entre eixos $3,6 \mathrm{~m}$.

Os resultados são apresentados nos itens a seguir. 
4.1. Teste de frenagem de $80 \mathrm{~km} / \mathrm{h}$ a $0 \mathrm{~km} / \mathrm{h}$ em pista com coeficiente de atrito de 0,3 utilizando a ECU ABS

As figuras 9 e 10 apresentam os gráficos das velocidades das rodas nos eixos dianteiro e traseiro, respectivamente. Em ambos os gráficos também se visualiza o valor da velocidade de referência do veículo.

É possível observar que à medida que a ECU ABS identifica a tendência de travamento das rodas, iniciam-se os ciclos de controle do módulo, o qual envia comandos aos solenoides das válvulas moduladoras para operarem conforme descrição da tabela 3 (Modos de operação de uma válvula moduladora ABS).

Os gráficos demonstram o controle eficiente realizado pela ECU, o qual evita o travamento das rodas durante todo o processo de frenagem. Tal aspecto corrobora o modelo matemático desenvolvido para as válvulas moduladoras.

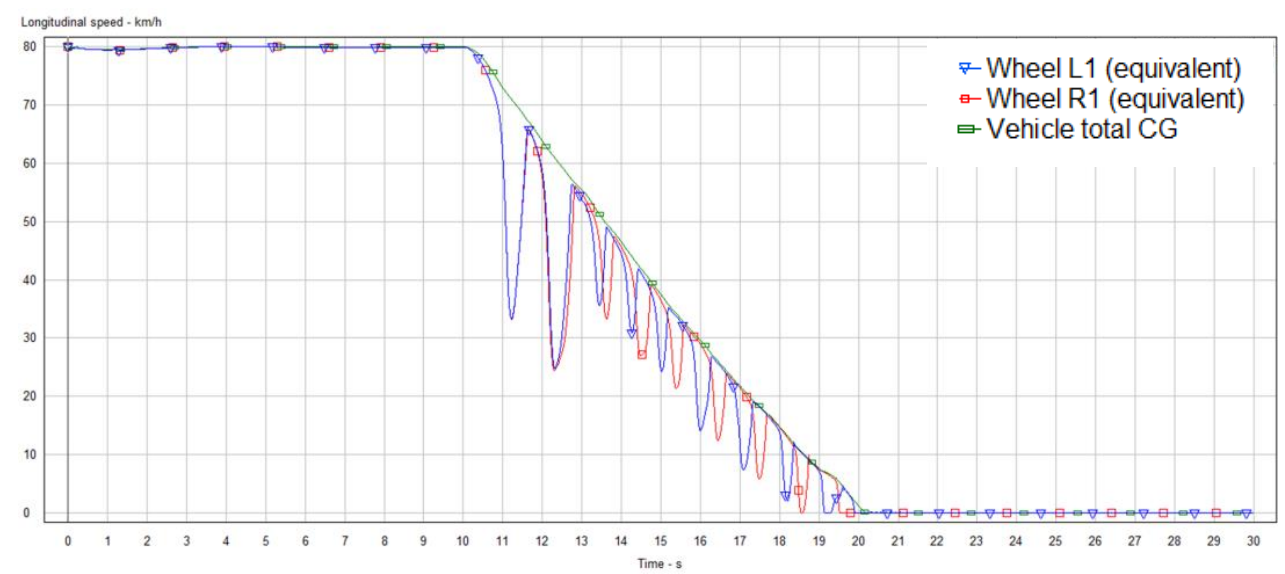

FIGURA 9. Velocidades das rodas do eixo dianteiro e velocidade de referência do veículo

FONTE: Software de simulação veicular

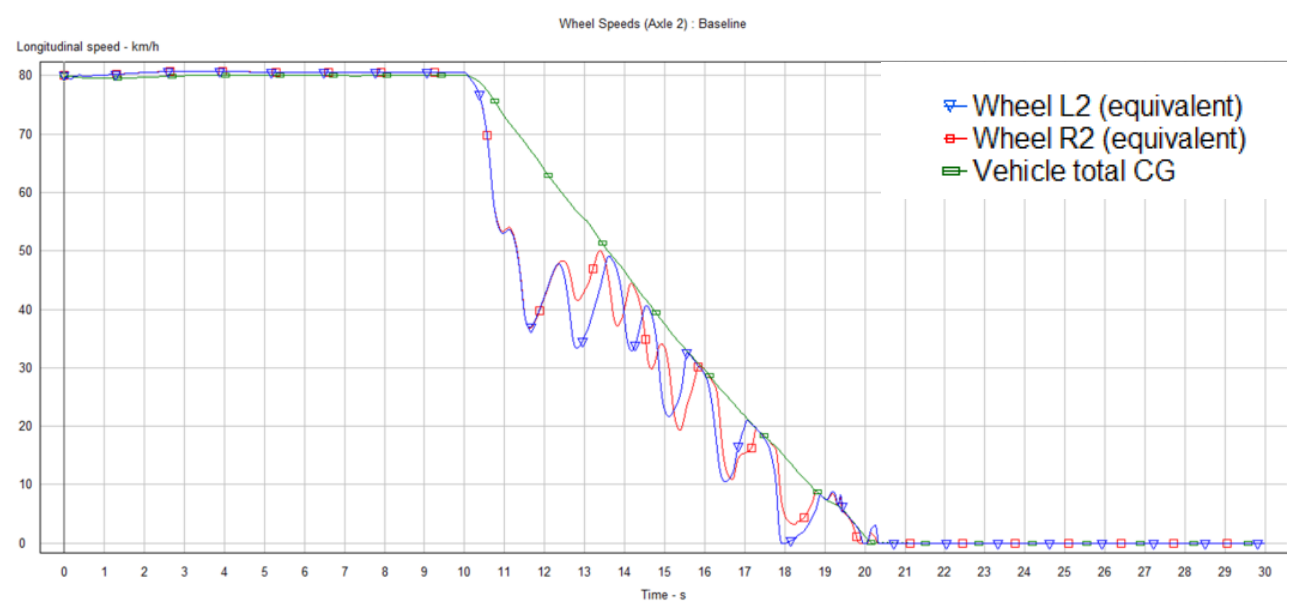

FIGURA 10. Velocidades das rodas do eixo traseiro e velocidade de referência do veículo

FONTE: Software de simulação veicular 
4.2. Teste de frenagem de $80 \mathrm{~km} / \mathrm{h}$ a $0 \mathrm{~km} / \mathrm{h}$ em pista com coeficiente de atrito de 0,3 sem ABS

As figuras 11 e 12 apresentam os gráficos das velocidades das rodas nos eixos dianteiro e traseiro, respectivamente. Em ambos os gráficos também se visualiza o valor da velocidade de referência do veículo.

É possível observar que sem o uso da ECU ABS na plataforma HIL, todas as rodas do veículo simulado travaram o que provoca o deslizamento e consequente perda do controle.

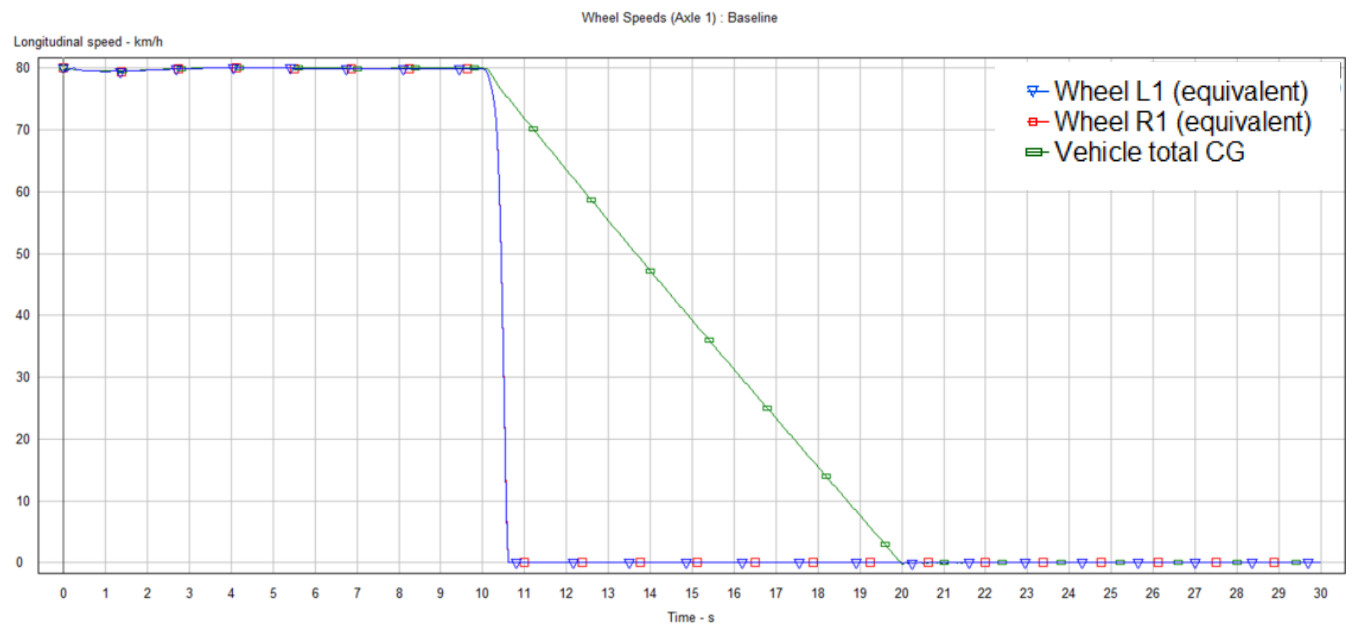

FIGURA 11. Velocidades das rodas do eixo dianteiro e velocidade de referência do veículo

FONTE: Software de simulação veicular

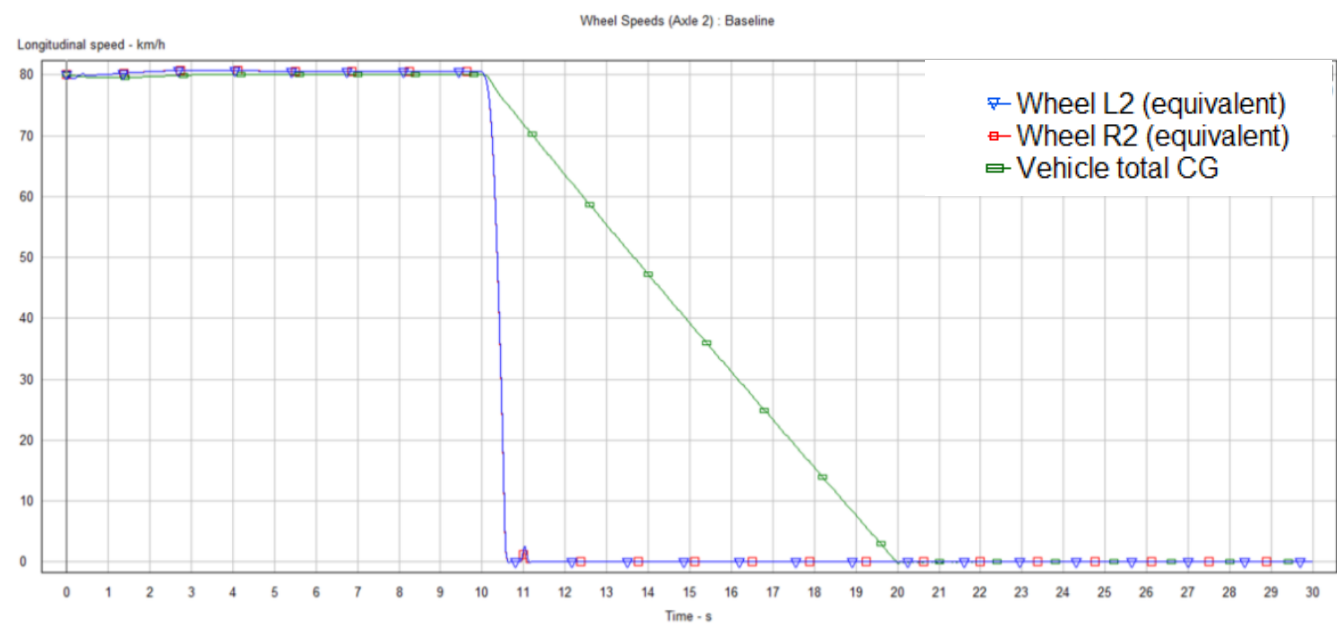

FIGURA 12. Velocidades das rodas do eixo traseiro e velocidade de referência do veículo

FONTE: Software de simulação veicular 
4.3. Teste de frenagem $\mu$-split de $50 \mathrm{~km} / \mathrm{h}$ a $0 \mathrm{~km} / \mathrm{h}$ com ABS

Nesta simulação foi realizado o teste de frenagem $\mu$-split com pista de coeficiente de atrito de 0,2 do lado esquerdo e de 0,5 do lado direito.

As figuras 13 e 14 apresentam os gráficos das velocidades das rodas nos eixos dianteiro e traseiro, respectivamente. Em ambos os gráficos também se visualiza o valor da velocidade de referência do veículo.

Neste caso também é possível observar a eficácia do sistema ABS evitando o travamento das rodas durante todo o processo de frenagem, o que reafirma a validade do modelo matemático desenvolvido para as válvulas moduladoras.

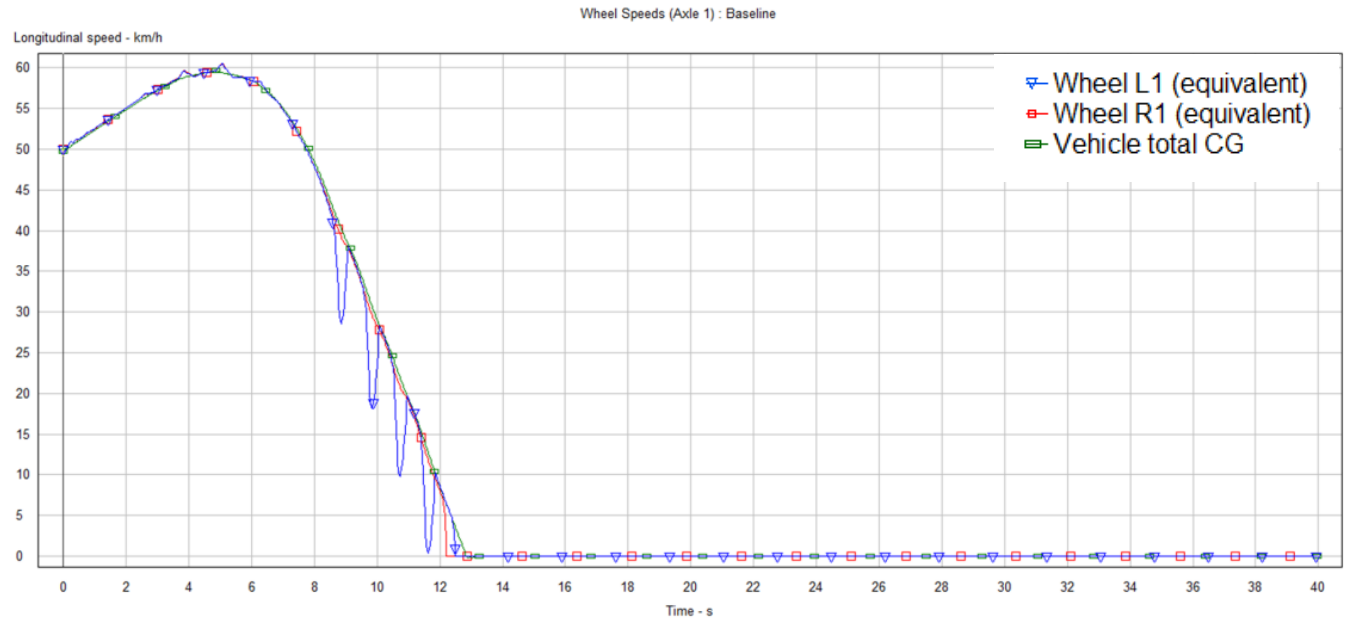

FIGURA 13. Velocidades das rodas do eixo dianteiro e velocidade de referência do veículo

FONTE: Software de simulação veicular

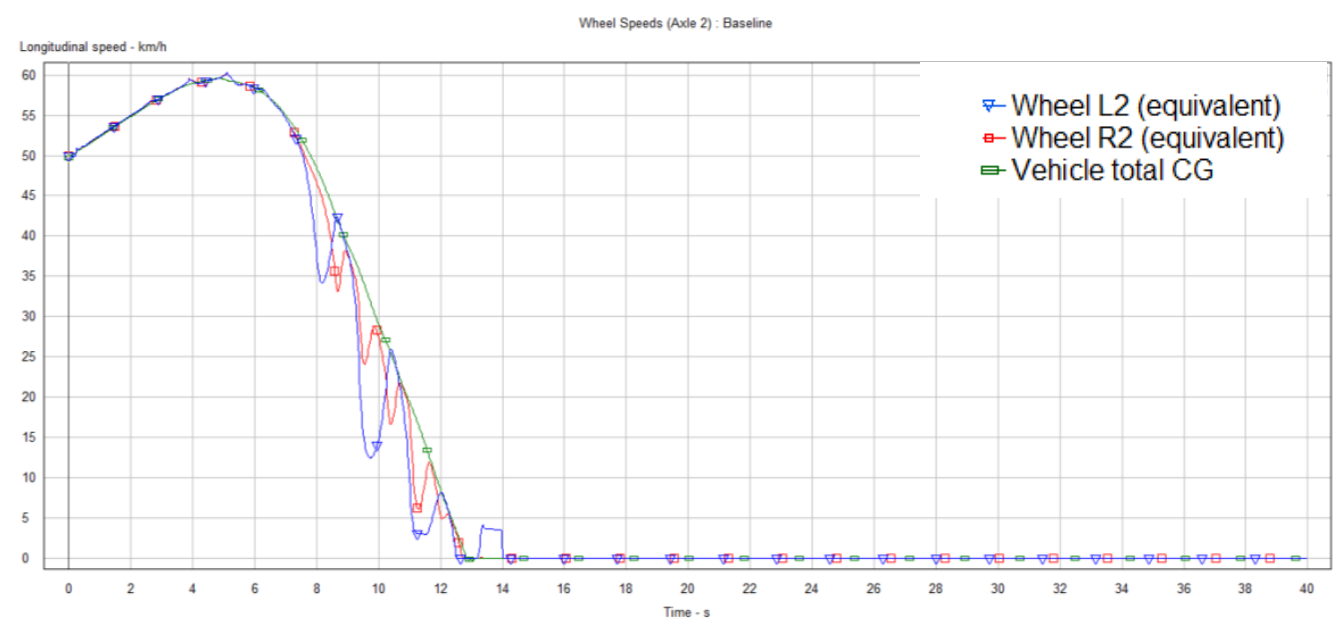

FIGURA 14. Velocidades das rodas do eixo traseiro e velocidade de referência do veículo

FONTE: Software de simulação veicular 
4.4. Teste de frenagem $\mu$-split de $50 \mathrm{~km} / \mathrm{h}$ a $0 \mathrm{~km} / \mathrm{h}$ sem ABS

Nesta simulação foi realizado o teste de frenagem com as mesmas condições de pista descritas no item 4.3.

As figuras 15 e 16 apresentam os gráficos das velocidades das rodas nos eixos dianteiro e traseiro, respectivamente. Em ambos os gráficos também se visualiza o valor da velocidade de referência do veículo.

É possível observar que sem o uso da ECU ABS na plataforma HIL, todas as rodas do veículo simulado travaram o que provoca o deslizamento e consequente perda do controle.

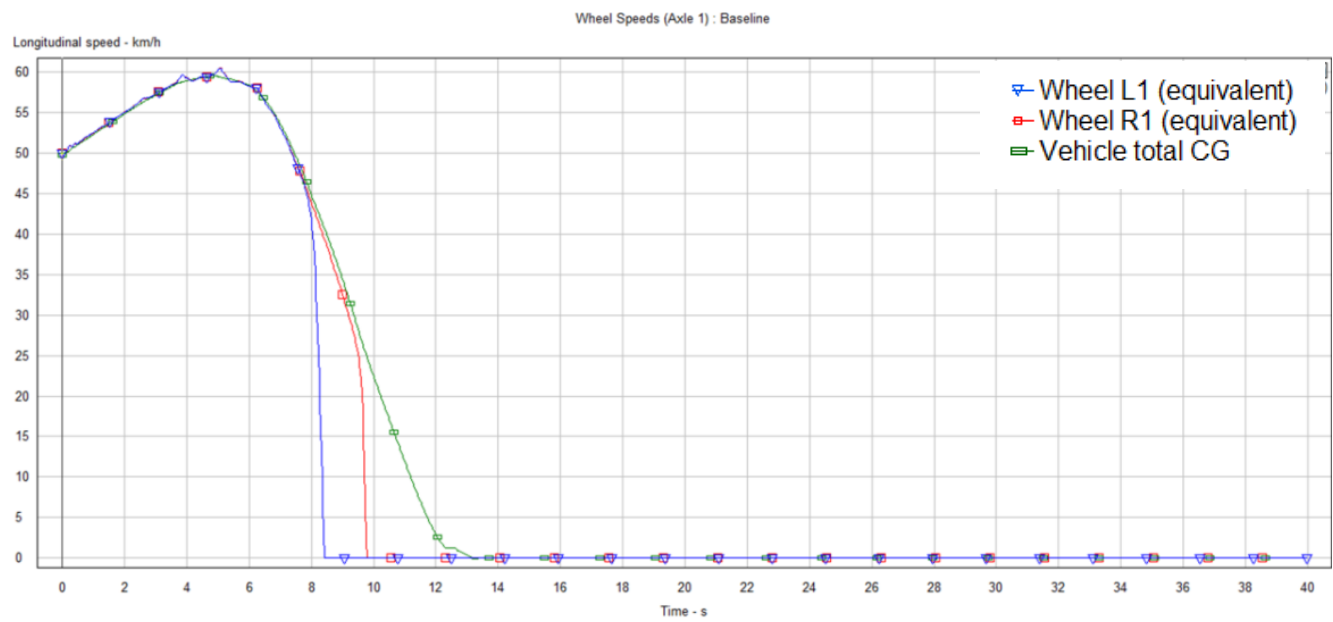

FIGURA 15. Velocidades das rodas do eixo dianteiro e velocidade de referência do veículo

FONTE: Software de simulação veicular

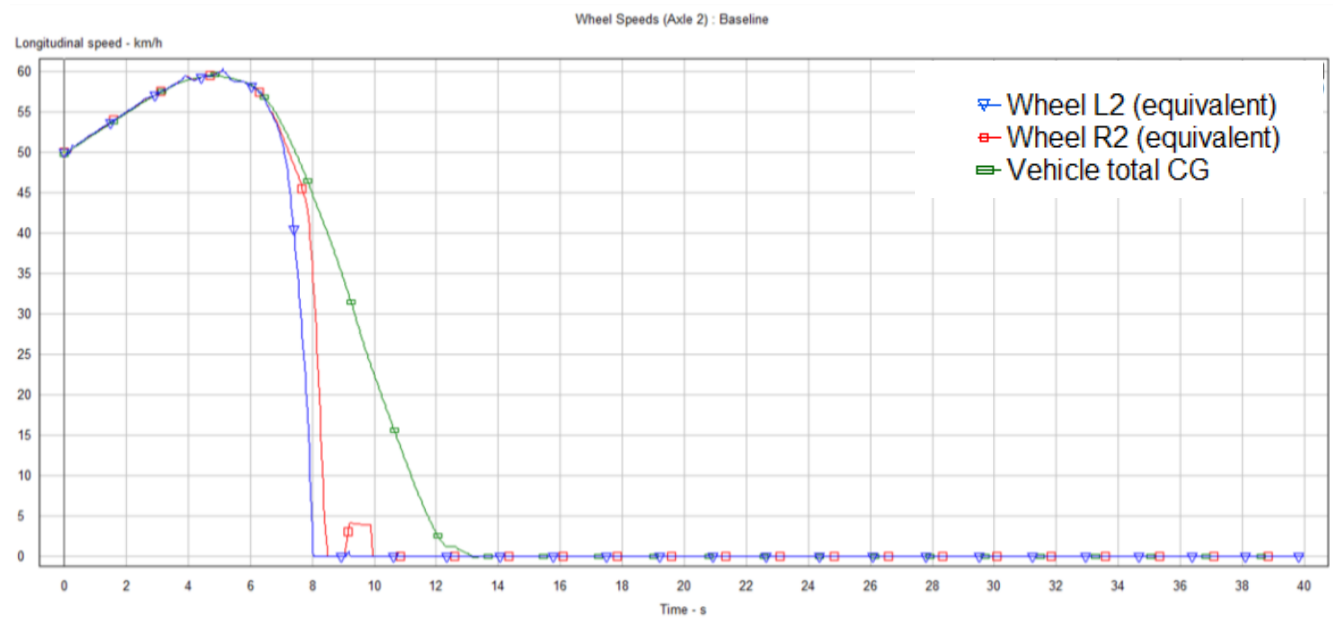

FIGURA 16. Velocidades das rodas do eixo traseiro e velocidade de referência do veículo

FONTE: Software de simulação veicular 


\section{CONCLUSÃO}

Este trabalho apresentou os detalhes de desenvolvimento de uma plataforma HIL de baixo custo de hardware aplicada à validação de uma ECU ABS.

A concepção do hardware para emulação de cargas e do software para produção de sinais e determinação de valores foram aspectos fundamentais para o êxito na operação da plataforma.

Os resultados foram obtidos através da realização de simulações executadas em diferentes condições de configuração do veículo (com e sem ABS) e condições da pista de teste, fatores que foram manipulados através da plataforma HIL.

A partir dos dados obtidos, foi possível visualizar de forma eficaz os efeitos produzidos pelo sistema ABS no controle da frenagem de um veículo, assim como, efetuar a comparação com testes realizados nas mesmas condições, porém sem o uso do sistema ABS.

$\mathrm{Na}$ construção do hardware desta plataforma foram utilizados componentes de baixo custo, aspecto que colabora para a ampliação do uso de simulação HIL no processo de validação de ECU's.

Além disso, o desenvolvimento apresentado neste trabalho abre a possibilidade para alavancar a produção deste tipo de tecnologia no mercado nacional e também sua eventual aplicação para fins instrutivos em ambiente acadêmico.

\section{REFERÊNCIAS}

[1] DELZANGLE, Craig. Embedded Systems in Automobiles. Em: <http://www.iup.edu/WorkArea/DownloadAsset.aspx?id=170652>. Acesso em: 01 Maio 2016.

[2] CHARETTE, R. N. This Car Runs On Code. New York: IEEE Spectrum; Em: <http://spectrum.ieee.org/green-tech/advanced-cars/this-car-runs-on-code>, 2009. Acesso em: 01 Maio 2016.

[3] "Networking the way to new systems", International Automobile Management, 1/2002, pp. 10-13.

[4] GREIMEL, Hans. Toyota recalls Prius models to update software. Automotive News. Em: <http://www.autonews.com/article/20140212/COPY01/302129954/toyota-recalls-priusmodels-to-update-software>. Acesso em: 01 Maio 2016.

[5] SANTOS, Max Mauro Dias; NEME, João Henrique Zander. A metodologia de desenvolvimento em modelos para software automotive: Hands-on. Disponível em : < sbesc.lisha.ufsc.br/sbesc2015/display2230>. Acesso em 22 Maio 2016.

[6] SILVA, Hilgad Montelo da. Simulação com hardware in the loop aplicada a veículos submarinos semi-autônomos. 2008. Tese de Mestrado. Universidade de São Paulo. 
[7] GÜHMANN, Clemens. "Model-based testing of automotive electronic control units." Test 2005 Conference, May. 2005.

[8] FATHY, H. et al. Review of hardware-in-the-loop simulation and its prospects in the automotive area. Ann Arbor, v. 1001, p. 48109-2125, 2006.

[9] TOBRO, Christian ; MAGNUSSON, Mathias. Improving wheel speed sensing and estimation. Department of Automatic Control. Lund Institut of Technology. 2003. 